\title{
On Selection Cooperation in Distributed Networks
}

\author{
Elzbieta Beres, and Raviraj Adve \\ University of Toronto \\ 10 King's College Road \\ Toronto, ON M5S 3G4, Canada
}

\begin{abstract}
We argue for Selection cooperation as an alternative to Distributed Space-Time Block Coded (DSTBC) transmissions and other Maximal Ratio Combining (MRC)-based schemes in cooperative wireless systems. While MRC is optimal in traditional receive diversity schemes, we show that in distributed networks, where transmitting nodes have a power, Selection cooperation outperforms MRC-based protocols. We present two different relay selection schemes based on available centralization and computing power and show that even the simplest selection scheme outperforms DSTBC in terms of outage probability.
\end{abstract}

\section{INTRODUCTION}

In distributed wireless systems, cooperative diversity and relaying can harness the advantages of multiple antennas without using multiple antennas on receivers and transmitters. For practical distributed networks this is motivated by the need for simple, inexpensive nodes with limited processing power and a single receive antenna. Additional antennas, if available, can be used to provide further performance gains.

Most available research on cooperative diversity has focused on a coherent addition of multiple independently-faded copies of the signal. The challenge in such a system is presented by the difficulty of sending multiple copies of the same data from distributed nodes. Several methods of implementing such a system have been proposed in the literature. In [1], Sendonaris et al. assume the relays know the phase of the relay-destination channel, they can pre-code before transmission such that the relay receives coherently added signals. This requires that terminals have accurate knowledge of the forward channel, and is thus difficult to implement. The authors also propose the use of a RAKE receiver in CDMA systems: multiple copies of the signal would be transmitted from all relays using the same spreading code, and the signals would be collected coherently with multiple fingers of a RAKE receiver. While practical, in spite of the increased complexity of the receiver, it limits relaying and cooperative diversity to CDMA systems.

Coherent combination can also be achieved through orthogonal transmissions and a maximal-ratio-combiner (MRC) at the receiver [2]-[4], or through distributed space-time codes (DSTC) [5]-[7]. In networks with several nodes, orthogonal transmissions incur a bandwidth penalty and maintaining the same rate forces an increase in spectral efficiency proportional to the number of relays. Switching to higher constellations, however, has a detrimental effect both on the outage probability and the BER performance, severely limiting the benefits of diversity [4], [7]. Implementations of DSTCs appear to be problematic since they require symbol and carrier synchronization between distant cooperating nodes.
These problems with the available protocols for distributed systems can be eliminated with use of Selection cooperation. In such a scheme, as with traditional selection diversity, after a data-sharing phase only the relay with the strongest channel to the destination would transmit. With $m-1$ relays, order- $m$ diversity could be achieved with only one additional orthogonal channel (required by the half-duplex constraint, which precludes nodes from receiving and transmitting on the same channel) as opposed to $m-1$ additional orthogonal channels in a MRC system implemented using orthogonal channels.

To date, the performance of Selection has not been examined in the literature on cooperative diversity. The available references in Selection have focused on network-layer issues [8], [9]. In some ways, diversity results can be directly carried over from traditional receive diversity - it is already understood that selection has the same diversity gain as MRC. Although in traditional receiver diversity systems MRC has a higher coding gain than Selection Combining, it is not clear apriori that this result would hold in cooperative systems, where each transmission requires a separate orthogonal channel and system performance can be affected by a per-node power constraint.

In this paper we show that, in the case of cooperative diversity, these considerations change the nature of the problem and make Selection cooperation a preferable choice to both orthogonal and space-time coded transmission. As explained above, the superior performance of Selection cooperation over multiple orthogonal transmissions is easily explained by the efficient use of bandwidth. The advantage over DSTC-systems, on the other hand, is gained not from bandwidth savings, as both schemes use the same amount of bandwidth, but from a more efficient use of power. In DSTC systems, each relay must share its available power between all source nodes; in a Selection cooperation system, a relay node divides its power only between the users that have chosen that node as a relay.

Because the power available at a relay node depends on the number of sources supported by that relay, relay selection becomes an interesting problem in the context of Selection cooperation. In this paper, we analyze two different relay selection schemes based on varying degrees of centralization and complexity tolerance, and show that both schemes outperform the DSTC system of [7].

This paper is structured as follows. Section II describes the system model and analyzes the Selection cooperation algorithm in a system with one source-destination pair. Section III 
presents two possible implementations of the scheme in a distributed network with multiple sources. Detailed derivations are delayed till the appendix. The main body of the paper focuses on the implementation issues and simulation results. Section IV concludes the work.

\section{SELECTION COOPERATION}

The distributed system comprises $m$ nodes communicating with their respective destinations. An in [7], we consider Decode-and-Forward schemes, although the results presented in the paper can easily be transferred to an Amplify-andForward scenario. The channel $a_{i j}$ between nodes $i$ and $j$ is modelled as flat and slowly-fading Rayleigh with variance $\lambda_{i j}$ and is independent of the channels. This model is appropriate for ad-hoc or mesh networks where each node has its own destination. Each node has an average power constraint of $P$ Joules/symbol.

We consider both a centralized and a non-centralized versions of the network. A centralized network is governed by a central unit $(\mathrm{CU})$ with knowledge of network parameters and the ability to make intelligent decisions based on this knowledge. In the absence of a $\mathrm{CU}$, the network is noncentralized and decisions are made locally by the nodes, with limited information regarding the rest of the network.

To introduce selection cooperation, consider a single source node $s$ communicating with a destination $d$ with the help of $m-1$ potential relays, $r_{1} \ldots r_{m-1}$. This communication uses a total bandwidth of $W \mathrm{~Hz}$. As in [7], relaying is performed in two-phases. In phase one, the source transmits information using $W / 2 \mathrm{~Hz}$. The destination and each of the $m-1$ relays decode this information. The decoding set $\mathcal{D}(s)$ for the source is the set of relays that decoded the information correctly, i.e., a node $r_{k} \in \mathcal{D}(s)$ if the capacity of the source-relay channel exceeds the required rate $R$ :

$$
\frac{1}{2} \log \left(1+\left|a_{s, r_{k}}\right|^{2} \mathrm{SNR}\right) \geq R,
$$

where the factor of $1 / 2$ models the bandwidth expansion required for relaying due to the half-duplex constraint, and $\mathrm{SNR}=P / N_{o}$ is the non-faded signal-to-noise ratio at a receiving node. The scheme of allowing only a subset of all nodes $m-1$ to relay is referred in [7] as Selection Relaying, and it is used to ensure full diversity order in decode-andforward schemes. We note here the potential ambiguity between the terms "Selection Relaying" and "Selection cooperation". While Selection Relaying ensures full-order diversity by forming a decoding set for each source, Selection cooperation restricts relaying to only one node from the decoding set.

In the second phase, each node in $\mathcal{D}(s)$ transmits a symbol to the destination identifying itself as a member of $\mathcal{D}(s)$. The destination (or the $\mathrm{CU}$ ) chooses a single relay with the best relay-destination channel to forward the information to the source using $W / 2 \mathrm{~Hz}$. Relaying is thus performed on orthogonal channels, but because only one relay is chosen for each source, the bandwidth expansion is only two-fold. As discussed in Section III, the choice of relay depends on the centralization in the network and on the tolerable complexity.

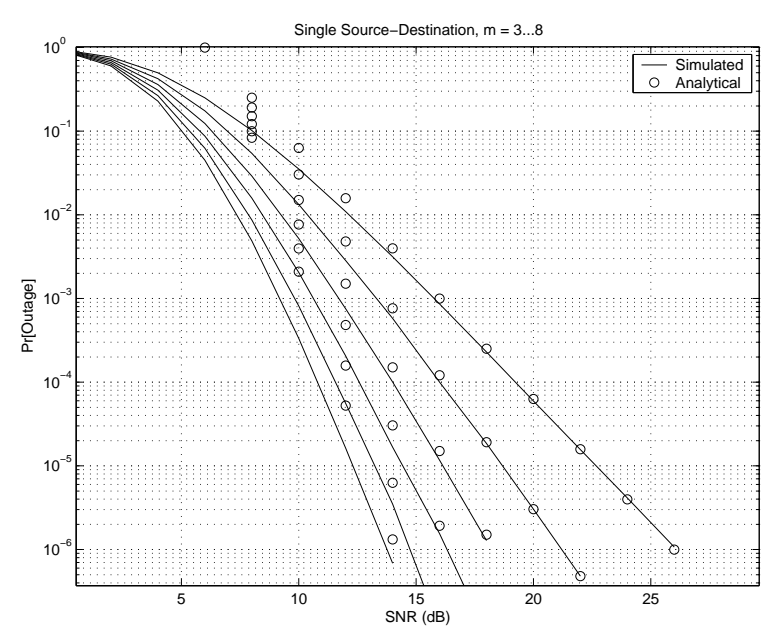

Fig. 1. Analytical and Simulated Outage Probability for Selection Cooperation with a single source-destination pair. $R=1 \mathrm{~b} / \mathrm{s} / \mathrm{Hz}, \lambda_{i j}=1, m=3 \ldots 8$

It is also worth mentioning that Selection cooperation does require some overhead not present in other MRC-based schemes. For every source transmission, all relays in the decoding set must transmit a signal informing the destination of their ability to relay. The destination or the $\mathrm{CU}$ must then transmit a signal to its selected relay allowing it transmit. All these transmissions must occur on orthogonal channels, decreasing the available bandwidth for the transmission and affecting system performance. We assume, however, that cooperation will be implemented in low-mobility environments where channels change slowly in time. In such a case, relays would not be changed often, and the overhead required would be minimal.

\section{A. Outage Probability}

The probability of outage, $P_{\text {out }}$, defined as the probability that the channel average mutual information $I_{\text {sel }}$ falls below the required rate $R$, is an important characterization of any cooperation protocol [7]. For the scenario described above, with $m-1$ potential nodes, the outage probability of the Selection scheme, in the high-SNR regime, is

$$
\begin{aligned}
P_{\text {out }} & =\left[\left(2^{2 R}-1\right) / \mathrm{SNR}\right]^{m} \lambda_{s, d} \sum_{|\mathcal{D}(s)|} \frac{1}{|\mathcal{D}(s)|+1} \\
& \times \prod_{r_{i} \in \mathcal{D}(s)} \lambda_{r_{i}, d} \prod_{r_{i} \notin \mathcal{D}(s)} \lambda_{s, r_{i}},
\end{aligned}
$$

where $1 / \lambda_{i j}$ is the average channel between node $i$ and $j$. The appendix provides the derivation of (2).

This approximation is verified in Figure 1 for increasing numbers of $m-1$ relays and for $\lambda_{i j}=1$ and $R=1 \mathrm{~b} / \mathrm{s} / \mathrm{Hz}$.. As expected, Selection cooperation exhibits full diversity order: with $m-1$ relays, we obtain order $m$ diversity. In the following section, this scheme is implemented in a network, and the results compared to those obtained in [7]. 


\section{NETWORK IMPLEMENTATION}

In this section, we extend the concept of Selection cooperation to network settings, using the notation of [7]. The network is composed of a set $\mathcal{M}$ of $m$ nodes. Each node $s \in \mathcal{M}$ has information to transmit to its own destination, $d(s) \notin \mathcal{M}$, and acts as a potential relay for other nodes in $\mathcal{M}$. We use the notation $s$ for a source node and $r$ for a relay node; we emphasize, however, that each node in $\mathcal{M}$ is a source node and each node has the potential of acting as a relay node.

As described in Section II, cooperation occurs in two phases. In phase one, all nodes use orthogonal channels to transmit information to their respective destinations, and each node decodes the information from the other $m-1$ nodes. For each source node $s_{i}$, a decoding set $\mathcal{D}\left(s_{i}\right)$ is formed from the nodes eligible to relay for node $s_{i}$. In phase two, a relay is chosen for each source $s_{i}$ from its decoding set $\mathcal{D}(s)$, and each relay forwards the information of the source. The activity of a node $s_{i}$ can thus be summarized as follows: in phase one, it transmits its information; in phase two, it decodes the information of the other $m-1$ nodes, and forwards the information of those nodes for which it was chosen as a relay.

A node potentially relaying for several others raises the question of power allocation. Each node has a discrete power constraint of $P$ Joules/symbol. In the first phase, each source sends its data using full power $P$ Joules/symbol. In the second phase, each relay divides its power evenly between the source nodes it is supporting. A relay node supporting $N$ source nodes will thus use $P / N$ Joules/symbol for each of the $N$ source nodes. Note that a relay node does not know a-priori how many nodes it will relay for and so cannot pre-compute a better power distribution. This is in contrast to the power allocation in the DSTBC scheme of [7], where every node always relays for all other $m-1$ nodes and thus uses $P / m$ Joules/symbol per source in both phases.

In this section we present two relay selection schemes based on varying degrees of centralization and tolerance of numerical complexity. The motivation between complex relay selection scheme is the effect of the per-node power constraint has on the network. Suppose that two source nodes, $s_{1}$ and $s_{2}$, are assigned to the same relay $r$, as it has the highest channel to $d\left(s_{1}\right)$ and $d\left(s_{2}\right)$. The power available at node $r$ for each source is $P / 2$. Better performance could potentially be achieved, however, by assigning one of the source nodes to a different "free" relay node with available power $P$. The problem is thus to assign relays to source nodes to minimize some figure of merit which depends on channel conditions as well as power available at the relays.

\section{A. Simple Relay Assignment}

The Simple Selection scheme is implemented in a system without centralization. The destination of $s_{i}, d\left(s_{i}\right)$ picks $r\left(s_{i}\right)$, the node with the highest relay-destination channel power, as the relay for source $s_{i}$ :

$$
r\left(s_{i}\right)=\arg \max _{r_{k} \in \mathcal{D}\left(s_{i}\right)}\left\{\left|a_{r_{k}}\right|^{2}\right\} ; \quad k=1 \ldots\left|\mathcal{D}\left(s_{i}\right)\right| .
$$

The scheme is of $O\left(\mathrm{~m}^{2}\right)$ complexity in the worst case, as each of the $m$ destinations must make $m-1$ comparisons to find a maximum out of (at most) $m-1$ channels. The scheme is also fully decentralized, as each destination picks its relay independently of all other nodes.

\section{B. Optimal Relay Assignment}

The mutual information of source $s_{i}$ using $r_{j}$ as a relay is:

$I_{s_{i} r_{j}}=\frac{1}{2} \log \left(1+\mathrm{SNR}\left|a_{s_{i}, d\left(s_{i}\right)}\right|^{2}+\frac{\mathrm{SNR}}{N_{j}}\left|a_{r_{j}, d\left(s_{i}\right)}\right|^{2}\right)$,

where $N_{j}$ is the number of source nodes for which node $j$ relays. One optimal algorithm calculates the mutual information of all $m$ users for all possible relay assignments, and picks the relay assignment which maximizes the minimum mutual information between the $m$ users:

$$
\begin{aligned}
& r\left(s_{1}\right), \ldots, r\left(s_{m}\right)= \\
& \quad \arg \max _{\forall i_{1} \in \mathcal{D}\left(s_{1}\right), \ldots i_{m} \in \mathcal{D}\left(s_{m}\right)} \min \left\{I_{s_{1} n_{i_{1}}}, \ldots I_{s_{m} n_{i_{m}}}\right\},
\end{aligned}
$$

where $I_{s_{2} n_{i_{2}}}$ denotes the mutual information between source $s_{2}$ and its destination $d\left(s_{2}\right)$, with node $n_{i_{2}}$, taken from $\mathcal{D}\left(s_{2}\right)$, used as a relay. This scheme is of $O\left(\mathrm{~m}^{m}\right)$ complexity, as it requires an exhaustive search through all potential $m^{m}$ relay combinations. The scheme is centralized, as it requires a $\mathrm{CU}$ with global knowledge to assign relays to sources.

\section{Outage Probability}

This section presents the analytical and simulated outage probabilities for the two schemes presented in Section II, and compares them to the outage probability of the distributed space-time protocols presented in [7]. The details of the derivations can be found in the Appendix.

Simple Selection Combining: With equal average channel powers with parameter $\lambda$, the probability of outage of the Simple Selection Scheme in the high-SNR regime is

$$
\begin{aligned}
& \operatorname{Pr}\left[I_{\text {simple-sel }}<R\right]= \\
& \quad\left[\left(2^{2 R}-1\right) / \mathrm{SNR}\right]^{m} \lambda^{m+1} \sum_{|\mathcal{D}(s)|} \frac{K_{|\mathcal{D}(s)|}(m)}{|\mathcal{D}(s)|+1},
\end{aligned}
$$

where

$$
K_{|\mathcal{D}(s)|}(m)=\sum_{N=1}^{m-1} \frac{\frac{N^{|\mathcal{D}(s)|}}{(m-N) ! N !(m-1)^{N}}}{\sum_{k=1}^{m-1} \frac{1}{(m-k) ! k !(m-1)^{k}}} .
$$

This approximation is verified in Figure 2, which compares the analytical and simulated results for the Simple Selection scheme. The analytical results are obtained by calculating (7) for increasing network sizes with $\lambda_{i j}=1$ and $R=1 \mathrm{~b} / \mathrm{s} / \mathrm{Hz}$.

As discussed in the appendix, the approximation assumes that the decoding set contains $m-1$ nodes for every source node, i.e., that every potential relay node correctly decodes the information from every source. The assumption is valid in the high SNR regime.

An analytical comparison of the DSTC and Simple Selection scheme is complicated by the difficulty in writing (7) with 


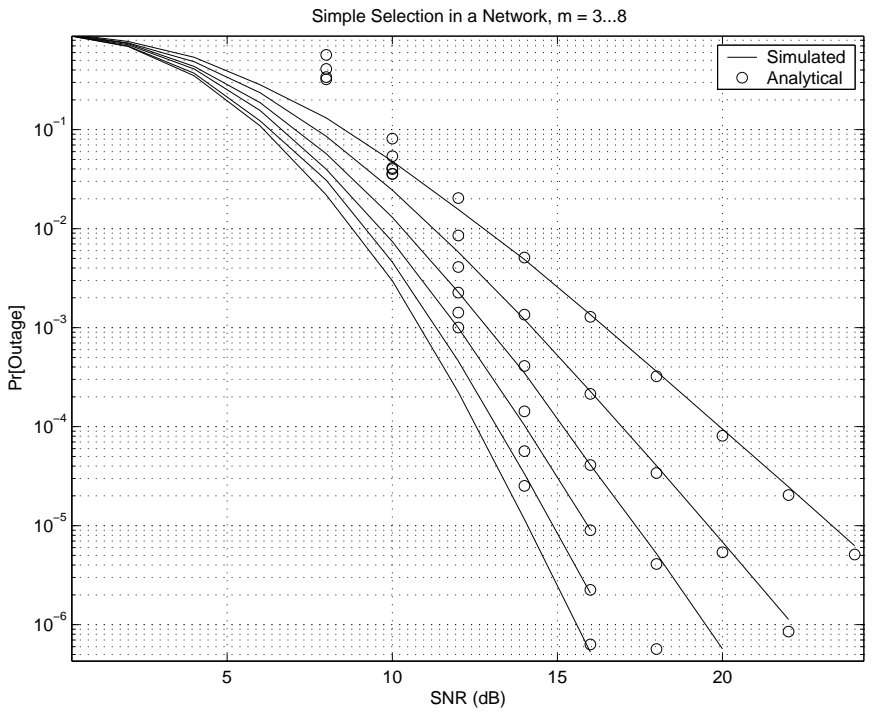

Fig. 2. Simple Selection Cooperation in a Network. $R=1 \mathrm{~b} / \mathrm{s} / \mathrm{Hz}, \lambda_{i j}=1$, $m=3 \ldots 8$

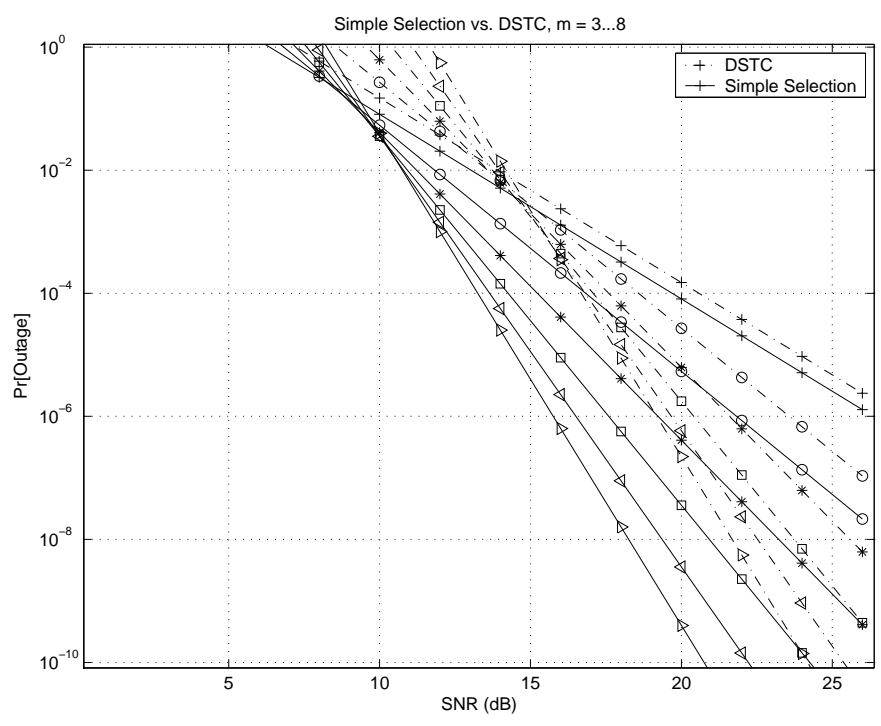

Fig. 3. Outage Probabilities of DSTC of [7] and Simple Selection Combining. $R=1 \mathrm{~b} / \mathrm{s} / \mathrm{Hz}, \lambda_{i j}=1, m=3 \ldots 8$

$m$ sources in closed-form. The comparisons are thus presented numerically in Figure 3 by calculating (7) and comparing to (22) in [7]. Note that the Simple Scheme always outperforms the DSTC scheme, and that the improvement increases for increasing $m$. The superior performance of Selection cooperation over an DSTC scheme might at first seem surprising and demonstrate the extent to which the distributed nature of cooperation changes the nature of the problem. In a distributed system where nodes have a power constraint, it is thus better to transmit using full-power from one node with the best channel than to transmit from all $m$ nodes with $1 / m$ th power.

Analytical results for the Optimal Selection scheme are difficult to obtain; in Figure 4 we present the outage probabil-

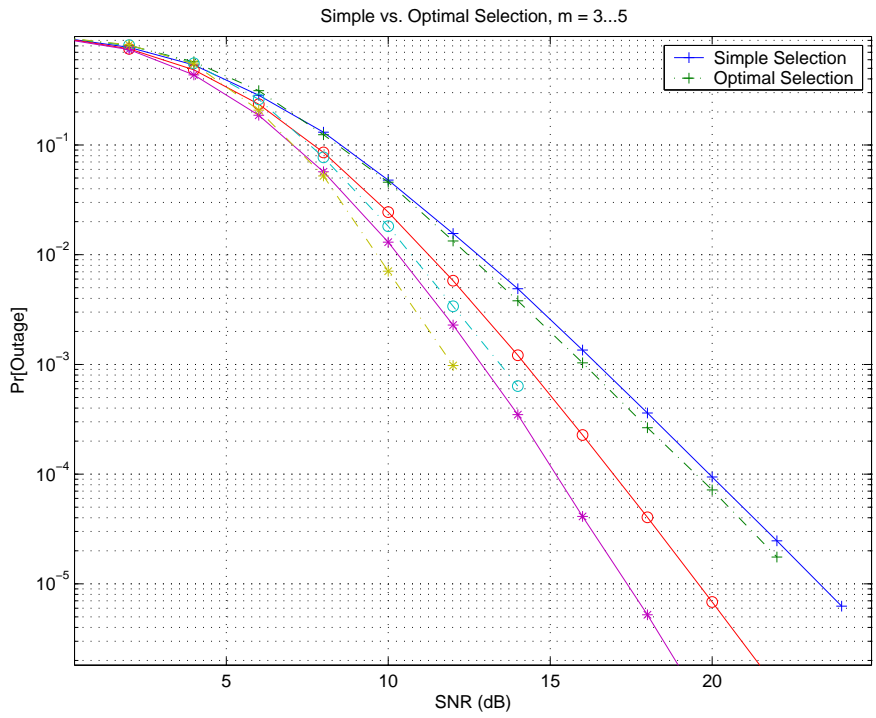

Fig. 4. Outage Probabilities of Simple and Optimal Selection $R=1 \mathrm{~b} / \mathrm{s} / \mathrm{Hz}$, $\lambda_{i j}=1, m=3 \ldots 5$

ity using simulations. Not surprisingly, performance improves with increasing system intelligence. We note, however, that the performance of the Simple scheme closely tracks that of the Optimal scheme for small network sizes, rendering the Simple Selection scheme a very good practical choice.

\section{Unequal Average Channels}

The result of (7) applies to cases where the average channels between all nodes are equal. Clearly, this is not the case in practical systems, where channel power is attenuated by distance and faded by shadowing. Although analytical results for such a case and general network sizes are difficult to obtain for the Simple Selection cooperation scheme, in Figure 5 we present simulations which compare the Simple Selection scheme to that the DSTC of [7] in this scenario. In this simulation, the channel power $\left|a_{i j}\right|^{2}$ is an exponential random variable with parameter $\lambda=e^{z}$, where $z$ is a zero mean normally distributed random variable with variance one.

The simulations demonstrate the improvement in performance of Simple Selection cooperation over DSTC. It is interesting to note that this improvement is higher than for the equal average channel case shown in Figure 3. This gap is explained by the fact that the performance of Simple Selection improves with increasing channel variation between nodes which decreases the probability of multiple relayed transmissions at relay nodes and thus increases the average power of relayed transmissions. We note that this is true only in a distributed scenario, where each node has a different destination.

\section{CONCLusions}

We have presented Selection cooperation as a practical alternative to distributed space-time coding. While providing 


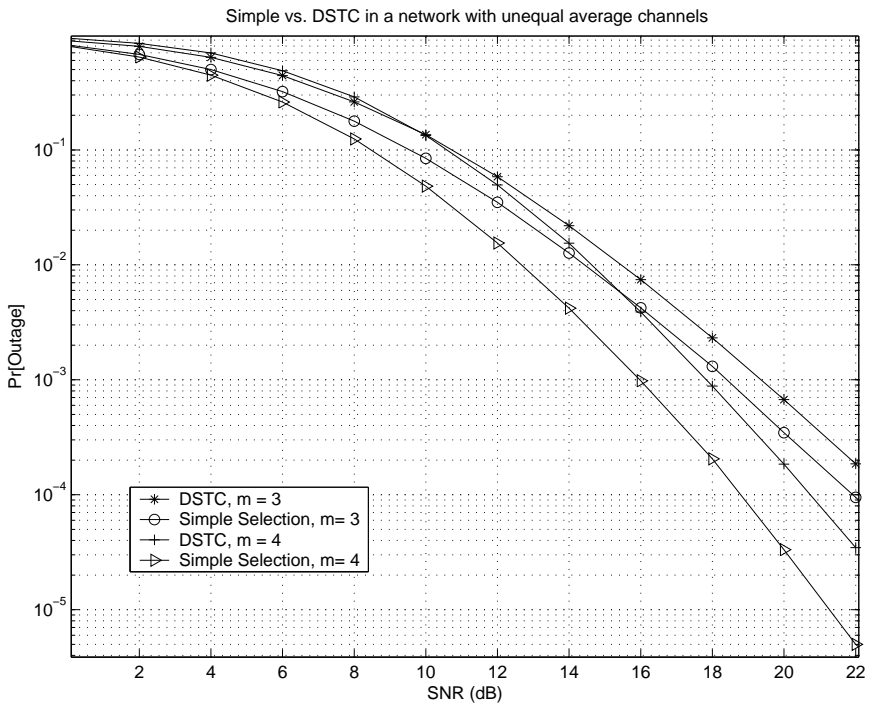

Fig. 5. Outage Probabilities of DSTC of [7] and Simple Selection with unequal average channels and $R=1 \mathrm{~b} / \mathrm{s} / \mathrm{Hz}, m=3,4$

the same diversity order, Selection cooperation does not require complex synchronization between relays. The price for Selection cooperation in a network setting is the overhead involved in choosing a relay; although this may have a small detrimental effect on the overall throughput, it does not change the straight-forward manner in which the scheme could be applied. Furthermore, neglecting the potential effects of bandwidth-loss due to overhead, both the Simple and Optimal Selection cooperation schemes significantly outperform DSTC in a distributed system, and the performance of the Simple scheme is close to that of the Optimal scheme.

\section{APPENDIX}

In this section, we develop the outage probability $\operatorname{Pr}\left[I_{\text {sel }}<\right.$ $R]$ for Selection cooperation in a single user system. An in [7], we use the total probability law:

$$
\operatorname{Pr}\left[I_{\text {sel }}<R\right]=\sum_{\mathcal{D}(s)} \operatorname{Pr}[\mathcal{D}(s)] \operatorname{Pr}\left[I_{\text {sel }}<R \mid \mathcal{D}(s)\right] \text {. }
$$

\section{A. Probability of the Decoding Set}

This derivation is given in [7] and is summarized here for the sake of completeness.

In our Selection cooperation scheme, the source transmits its information using full power $P$. Nodes are in the decoding set if their channel from the source satisfies

$$
\begin{aligned}
\operatorname{Pr}[r \in \mathcal{D}(s)] & =\operatorname{Pr}\left[\left|a_{s, r}\right|^{2}>\left(2^{2 R}-1\right) / \mathrm{SNR}\right] \\
& =\exp \left[-\lambda_{s, r}\left(2^{2 R}-1\right) / \mathrm{SNR}\right]
\end{aligned}
$$

Because each relay makes independent decisions and the channel fading realizations are independent, the decoding set probability is

$$
\begin{aligned}
\operatorname{Pr}[\mathcal{D}(s)] & =\prod_{r \in \mathcal{D}(s)} \exp \left[-\lambda_{s, r}\left(2^{2 R}-1\right) / \mathrm{SNR}\right] \\
& \times \prod_{r \notin \mathcal{D}(s)}\left(1-\exp \left[-\lambda_{s, r}\left(2^{2 R}-1\right) / \mathrm{SNR}\right]\right) \\
& =\left[\frac{2^{2 R}-1}{\mathrm{SNR}}\right]^{m-\mathcal{D}(s)-1} \times \prod_{r \notin \mathcal{D}(s)} \lambda_{s, r}
\end{aligned}
$$

\section{B. Outage Probability conditioned on the Decoding Set}

In this section, we develop the outage probability for a single source node $s$ communicating with destination $d$ using a relay chosen among a maximum of $m-1$ relays from the decoding set.

We begin by defining the random variables $X$ and $Y$ as

$$
\begin{aligned}
Y & =\left|a_{s, d}\right|^{2} \\
f_{Y}(y) & =\lambda_{s d} \exp \left[-\lambda_{s d} y\right] \\
X & =\max _{r_{i} \in \mathcal{D}(s)}\left\{\left|a_{r_{1}, d}\right|^{2},\left|a_{r_{2}, d}\right|^{2}, \ldots,\left|a_{r_{N}, d}\right|^{2}\right\} \\
F_{X}(x) & =\prod_{i=1}^{|\mathcal{D}(s)|}\left(1-\exp \left[-N \lambda_{r_{i} d} y\right]\right)
\end{aligned}
$$

where the cumulative distribution function of $X$ in (14) is derived using the independence of $\left|a_{r_{i}, d(s)}\right|^{2} \forall i$. The channel mutual information is thus

$$
I_{\text {sel }}=\frac{1}{2} \log (1+\operatorname{SNR}(X+Y)) .
$$

Using approximation $e^{x} \sim(1+x)$ followed by the Binomial Expansion, we obtain the following, where $b$ denotes $\left(2^{2 R}-1\right) / \mathrm{SNR}$ :

$$
\begin{aligned}
& \Rightarrow \operatorname{Pr}\left[I_{\text {sel }}<R \mid \mathcal{D}(s)\right] \\
& =\operatorname{Pr}[(X+Y)<b] \\
& =\int_{0}^{b} \prod_{i=1}^{|\mathcal{D}(s)|}\left(1-\exp \left[-\lambda_{r_{i}, d} y\right]\right) \lambda_{s, d} \exp \left[-\lambda_{s, d} y\right] d y \\
& =\lambda_{s, d} \prod_{i=1}^{|\mathcal{D}(s)|} \lambda_{r_{i}, d} \int_{0}^{b}(b-y)^{|\mathcal{D}(s)|}\left(1-\lambda_{s, d} y\right) d y \\
& =\lambda_{s, d} \prod_{i=1}^{|\mathcal{D}(s)|} \lambda_{r_{i}, d} \\
& \times \int_{0}^{b} \sum_{j=0}^{|\mathcal{D}(s)|}\left(\begin{array}{c}
|\mathcal{D}(s)| \\
j
\end{array}\right) b^{|\mathcal{D}(s)|-j}(-y)^{j}\left(1-\lambda_{s, d} y\right) d y
\end{aligned}
$$

where $\left(\begin{array}{l}n \\ p\end{array}\right)=\frac{n !}{p !(n-p) !}$. After solving the integral, some manipulations and the use of identity 0.155 from [10], this expression reduces to

$$
\begin{array}{r}
b^{|\mathcal{D}(s)|+1} \lambda_{s, d} \times \sum_{j=0}^{|\mathcal{D}(s)|}\left(\begin{array}{c}
|\mathcal{D}(s)| \\
j
\end{array}\right) \frac{(-1)^{j}}{j+1} \prod_{i=1}^{|\mathcal{D}(s)|} \lambda_{r_{i}, d} \\
=b^{|\mathcal{D}(s)|+1} \lambda_{s, d} \frac{1}{|\mathcal{D}(s)|+1} \prod_{i=1}^{|\mathcal{D}(s)|} \lambda_{r_{i}, d} .
\end{array}
$$




\section{Outage Probability}

The total outage probability is obtained by substituting (10) and (16) into (8)

$$
\begin{aligned}
\operatorname{Pr}\left[I_{\text {sel }}<R\right] & =b^{m} \lambda_{s, d} \sum_{|\mathcal{D}(s)|} \frac{1}{|\mathcal{D}(s)|+1} \\
& \times \prod_{r_{i} \in \mathcal{D}(s)} \lambda_{r_{i}, d} \prod_{r_{i} \notin \mathcal{D}(s)} \lambda_{s, r_{i}} \\
& =\left[\left(2^{2 R}-1\right) / \mathrm{SNR}\right]^{m} \lambda_{s, d} \sum_{|\mathcal{D}(s)|} \frac{1}{|\mathcal{D}(s)|+1} \\
& \times \prod_{r_{i} \in \mathcal{D}(s)} \lambda_{r_{i}, d} \prod_{r_{i} \notin \mathcal{D}(s)} \lambda_{s, r_{i}} .
\end{aligned}
$$

\section{Outage Probability of Simple Selection}

In this section, we develop the outage probability bounds Simple Selection cooperation in network setting. We begin with $\operatorname{Pr}\left[I_{\text {sel }}<R \mid N\right]$, the outage probability of a source node $s \in \mathcal{M}$ communicating with destination $d$ using a relay already supporting a total of $N$ source nodes and thus expanding $P / N$ Joules/symbol for each supported source node. The mutual information is thus

$$
I_{\text {sel }}=\frac{1}{2} \log \left(1+\mathrm{SNR} \times Y+\frac{\mathrm{SNR}}{N} X\right),
$$

where $Y$ and $f_{Y}(y)$ are defined in (11) and (12) and

$$
\begin{aligned}
X & =\frac{1}{N} \max _{r_{i} \in \mathcal{D}(s)}\left\{\left|a_{r_{1}, d}\right|^{2},\left|a_{r_{2}, d}\right|^{2}, \ldots,\left|a_{r_{N}, d}\right|^{2}\right\}, \\
F_{X}(x) & =\prod_{i=1}^{|\mathcal{D}(s)|}\left(1-\exp \left[-N \lambda_{r_{i} d} y\right]\right) .
\end{aligned}
$$

The development of $\operatorname{Pr}\left[I_{\text {sel }}<R \mid N\right]$ is very similar to that shown in Sections B and C and we thus give only the final result:

$$
\begin{aligned}
\operatorname{Pr}\left[I_{\text {sel }}<R \mid N\right] & =\left[\left(2^{2 R}-1\right) / \mathrm{SNR}\right]^{m} \lambda_{s, d} \sum_{|\mathcal{D}(s)|} \frac{N^{|\mathcal{D}(s)|}}{|\mathcal{D}(s)|+1} \\
& \times \prod_{r_{i} \in \mathcal{D}(s)} \lambda_{r_{i}, d} \prod_{r_{i} \notin \mathcal{D}(s)} \lambda_{s, r_{i}} .
\end{aligned}
$$

The outage probability of Simple cooperation can now be obtained by again using the total probability law

$$
\operatorname{Pr}\left[I_{\text {sel }}<R\right]=\sum_{N=1}^{m-1} \operatorname{Pr}\left[I_{\text {sel }}<R \mid N\right] p_{N}(n),
$$

where we use $p_{N}(n)$ to denote the probability of a relay supporting $N$ source nodes, where we exclude the possibility of a source relaying for itself, and thus $0 \leq N \leq m-1$. In the case of equal average channels, this probability can be approximated in the high SNR region as

$$
p_{N}(n)=\left(\begin{array}{c}
m-1 \\
n
\end{array}\right)\left[\frac{1}{m-1}\right]^{n}\left[\frac{m-2}{m-1}\right]^{m-1-n}
$$

We are only interested, however, in the probability of a node supporting $n$ sources, given that it has already been chosen as a relay and is thus supporting at least one source, i.e., $p(N=$ $n \mid N \geq 1$ ). Using Bayes' rule, we obtain

$$
\begin{aligned}
p(N & =n \mid N \geq 1)=\frac{p\{(N=n) \&(n \geq 1)\}}{p\{N \geq 1\}} \\
& =\frac{p(N=n)}{\sum_{k=1}^{m-1} p(N=k)}, \quad \forall n=1 \ldots m-1 \\
& =\frac{\left(\begin{array}{c}
m-1 \\
N
\end{array}\right)\left[\frac{1}{m-1}\right]^{N}\left[\frac{m-2}{m-1}\right]^{m-1-N}}{\sum_{k=1}^{m-1}\left(\begin{array}{c}
m-1 \\
k
\end{array}\right)\left[\frac{1}{m-1}\right]^{k}\left[\frac{m-2}{m-1}\right]^{m-1-k}}, \quad \forall n=1 \ldots m-1,
\end{aligned}
$$

We note that the approximation of (23), valid for high signalto-noise scenarios, assumes that the relay belongs to the decoding set of every source. Combining (20), (21) and (23) results in:

$$
\begin{array}{r}
\operatorname{Pr}\left[I_{\text {simple-sel }}<R\right]=\left[\left(2^{2 R}-1\right) / \mathrm{SNR}\right]^{m} \lambda^{m+1} \\
\times \sum_{|\mathcal{D}(s)|} \frac{K_{|\mathcal{D}(s)|}(m)}{|\mathcal{D}(s)|+1},
\end{array}
$$

where

$$
K_{|\mathcal{D}(s)|}(m)=\sum_{N=1}^{m-1} \frac{\frac{N^{|\mathcal{D}(s)|}}{(m-N) ! N !(m-1)^{N}}}{\sum_{k=1}^{m-1} \frac{1}{(m-k) ! k !(m-1)^{k}}} .
$$

\section{REFERENCES}

[1] A. Sendonaris, E. Erkip, and B. Aazhang, "User cooperation diversity part I, II," IEEE Transactions on Communications, vol. 51, pp. 1927 1948, November 2003.

[2] J. N. Laneman, D. N. C. Tse, and G. W. Wornell, "Cooperative diversity in wireless networks: Efficient protocols and outage behavior,' IEEE Transactions on Information Theory, vol. 50, pp. 3062 - 3080, December 2004.

[3] J. Boyer, D. Falconer, and H. Yanikomeroglu, "A resource allocation strategy for distributed MIMO multi-hop communication systems," IEEE Transactions on Communications, vol. 52, pp. 1820 - 1830, October 2004.

[4] P. Herhold, E. Zimmermann, and G. Fettweis, "Co-operative multihop transmission in wiresless networks," Computer Networks Journal, Submitted 2004

[5] S. Barbarossa and G. Scutari, "Distributed space-time coding for multihop networks," in Proceedings of IEEE International Conference on Communications, ICC 2004, June 2004. Paris, France.

[6] M. Uysal and O. Canpolat, "On the distributed space-time signal design for a large number of relay terminals," in Proceedings of IEEE Wireless Communications and Networking Conference, March 2005.

[7] J. N. Laneman and G. W. Wornell, "Distributed space-time-coded protocols for exploiting cooperative diversity in wireless networks," IEEE Transactions on Information Theory, vol. 49, pp. 2415 - 2425, October 2003

[8] S. Hares, H. Yanikomeroglu, and B. Hashem, "A relaying algorithm for multihop tdma tdd networks using diversity," in Proceedings of IEEE Vehicular Technology Conference, October 2003.

[9] P. Larsson and N. Johansson, "Multiuser diversity forwarding in multihop packet radio networks," in Proceedings of IEEE Wireless Communications and Networking Conference, March 2005.

[10] I. S. Gradshteyn, I. M. Ryzhik, and A. J. (Ed), Table of Integrals, Series and Products. Academic Press, 1984. 